\title{
Avances en el Estudio de la Diversidad Bacteriana Oral Asociada a Caries Dental Mediante el Estudio Genómico
}

\author{
Advances in the Study of Oral Bacterial Diversity \\ Associated with Dental Caries by Genomic Study
}

\begin{abstract}
Bárbara Astorga*; Constanza Barraza*; Juan Manuel Casals*; María José Cisterna*; Daniela Mena*; Francisca Morales ${ }^{*}$ S Sergio González ${ }^{* *}$ Osmir de Oliveira Junior ${ }^{* * *}$ \& Gustavo Moncada $^{\star \star * *}$
\end{abstract}

ASTORGA, B.; BARRAZA, C.; CASALS, J. M.; CISTERNA, M. J.; MENA, D.; MORALES, F.; GONZÁLEZ, S.; OLIVEIRA JUNIOR, O. \& MONCADA, G. Avances en el estudio de la diversidad bacteriana oral asociada a caries dental mediante el estudio genómico. Int. J. Odontostomat., 9(3):349-356, 2015.

RESUMEN: La caries es una enfermedad infecciosa, transmisible y multifactorial, que conduce a la pérdida de minerales reversible o irreversible de los tejidos duros susceptibles del diente, por acción de productos ácidos provenientes de la fermentación de los hidratos de carbono de la dieta por la actividad metabólica del biofilm adherido a la superficie dentaria. Aunque tradicionalmente se ha considerado al Streptococcus mutans como el responsable de la enfermedad, actualmente otras bacterias, denominadas no mutans, se han asociado con el inicio, progresión y actividad de la enfermedad en esmalte, dentina y cemento radicular. Para profundizar el estudio de la diversidad bacteriana oral asociada a caries dental se han aplicado diversas metodologías, dentro de las cuales destaca el estudio del metagenoma oral. Este nos permite estudiar comunidades bacterianas completas mediante el análisis del DNA, en un determinado ambiente sin necesidad de aislar y cultivar las especies, entregando información sobre la diversidad taxonómica y filogenética de estas comunidades. Existen diferentes métodos de análisis de la diversidad bactariana, entre los que tenemos el análisis del ARNr 16S mediante electroforésis, PCR, microarreglos, secuenciamiento de última generación, entre otros. El estudio del metagenoma oral ha permitido identificar especies que no han podido ser aisladas por métodos convencionales, además de identificar su presencia o ausencia en las distintas etapas del desarrollo de la enfermedad de caries dental, permitiendo un mejor conocimiento del desarrollo de esta patología. El estudio basado en el metagenoma ha dado a conocer una diversidad microbiana oral inesperada, dando información relevante para la actualización de los conocimientos y así identificar nuevos objetivos terapéuticos. El propósito de esta revisión bibliográfica es exponer los principales resultados que ha aportado el estudio del metagenoma sobre la diversidad microbiana, aplicado específicamente a la comunidad bacteriana oral.

PALABRAS CLAVE: caries dental, metagenoma, microbioma oral, ARNs $16 \mathrm{~S}$.

\section{INTRODUCCIÓN}

La caries es una enfermedad infecciosa, transmisible y multifactorial, que conduce a la pérdida de minerales reversible o irreversible de los tejidos duros susceptibles del diente, por acción de productos ácidos provenientes de la fermentación de los hidratos de carbono de la dieta por la actividad metabólica del biofilm adherido a la superficie dentaria (Fontana et al., 2010). La caries dental es un proceso dinámico que se produce por un desequilibrio entre la remineralización y la desmineralización del diente. La participación bacteriana en la etiología de la caries se ha explicado mediante tres hipótesis, la primera es la placa no específica propuesta por Theilade en 1986, la segunda es la placa específica propuesta por Loesche en 1992 y la tercera es la placa ecológica propuesta por Marsh en 1994, siendo esta última la más aceptada (Aas et al., 2008).

Aunque tradicionalmente se ha considerado al Streptococcus mutans como el responsable de la en-

\footnotetext{
Alumno Pregrado, Escuela de Odontología, Universidad Mayor, Santiago, Chile.

* Profesor Asociado, Magíster en Cs. Biológicas, Asignatura de Cariología, Escuela de Odontología, Universidad Mayor, Santiago, Chile.

*** PhD, Departamento de Odontología restauradora, Universidade Estadual Paulista, UNESP, Araraquara, Brasil.

**** Profesor Titular, Decano Escuela de Odontología, Universidad Mayor, Santiago, Chile.
} 
ASTORGA, B.; BARRAZA, C.; CASALS, J. M.; CISTERNA, M. J.; MENA, D.; MORALES, F.; GONZÁLEZ, S.; OLIVEIRA JUNIOR, O. \& MONCADA, G. Avances en el estudio de la diversidad bacteriana oral asociada a caries dental mediante el estudio genómico. Int. J. Odontostomat., 9(3):349-356, 2015.

fermedad, actualmente otras bacterias, denominadas no mutans, se han asociado con el inicio, progresión y actividad de la enfermedad en esmalte, dentina y cemento radicular. Estas se han vinculado con el desequilibrio entre microorganismos acidogénicos y alcalinogénicos en el medio oral enriqueciendo la diversidad de bacterias involucradas en el proceso de caries dental (Aas et al., 2008).

Para profundizar el estudio de la diversidad bacteriana oral asociada a caries dental se han aplicado diversas metodologías, dentro de las cuales destaca el estudio del metagenoma oral. Este nos permite estudiar comunidades bacterianas completas mediante el análisis del DNA, en un determinado ambiente sin necesidad de aislar y cultivar las especies, entregando información sobre la diversidad taxonómica y filogenética de estas comunidades (Belda-Ferre et al., 2012). Este y otros importantes avances que se han alcanzado durante la última década, han contribuido a mejorar la comprensión de la etiopatogenia de la enfermedad y podrían modificar en el futuro el diagnóstico, prevención, manejo y pronóstico de la caries dental.

El propósito de esta revisión bibliográfica es exponer los principales resultados que ha aportado el estudio del metagenoma sobre la diversidad microbiana, aplicado específicamente a la comunidad bacteriana oral.

EI Proceso de Caries dental. La caries dental es la enfermedad crónica más frecuente en la especie humana. Su progreso es lento en la mayoría de los individuos, otorgando entre 3 a 4 años para la intervención preventiva antes de la cavitación en lesiones de caries proximales de molares permanentes (Shwartz et al., 1984; Hintze, 2011; Pitts, 1983).

La progresión de la lesión puede presentarse desde la pérdida neta de minerales por presencia de ácidos a nivel ultraestructural, hasta la destrucción total del diente. El desarrollo de la lesión de caries es un proceso dinámico con períodos alternados de progresión, detención y regresión (Nyvad et al., 2013), donde la placa dental juega un rol primordial.

La placa dental está definida como una comunidad bacteriana fuertemente adherida a la superficie dentaria, organizada, jerarquizada en sus funciones, adaptable al medio, de cooperación entre microorganismos, activa, clasificada según su localización en, supragingival y subgingival. La placa supragingival contiene flora predominantemente Gram positiva, comúnmente asociada a microorganismos cariogénicos (microorganimos acidogénicos y acidofílicos) y la placa subgingival, está compuesta en mayor cantidad de microorganismos anaerobios Gram negativos, que son preferentemente periodontopatogénicos (Seif et al., 1997).

Para explicar la participación de las bacterias en la etiología de caries dental se han propuesto tres hipótesis, las que abordan la composición y acción de la placa. La hipótesis de la placa no específica postula que la caries se forma mediante la actividad de la placa oral, donde todos los microorganismos que colonizan la superficie dentaria participan por igual en los procesos patológicos, siendo más importante la cantidad que el tipo de especies presentes. La hipótesis de la placa específica propone que determinadas especies, como el S. mutans y S. sobrinus están relacionados activamente con la enfermedad, siendo el efecto patogénico de la placa dependiente del tipo específico de microorganismo residente en ella. Por último, la placa ecológica postula que la presencia de ciertos patógenos de la placa bacteriana darían origen a enfermedades periodontales y caries dental, pero estaría influenciada por la condición del hospedero y factores ambientales como $\mathrm{pH}$, potencial redox, dieta $\mathrm{e}$ higiene (Aas et al., 2008).

Es conocido que la actividad metabólica de la placa dental genera ácidos débiles, que se pueden relacionar directamente con la pérdida neta de minerales. Por ejemplo en lesiones de esmalte superficial, la placa bacteriana contiene Streptococcus no mutans y Actinomyces, entre otros, que producen un ambiente de acidificación leve, fenómeno compatible con el equilibrio de la desmineralización/remineralización. Además de la producción de ácido, algunas bacterias como $S$. oralis, $S$. salivarius y $S$. gordonii pueden elevar el $\mathrm{pH}$ mediante la producción de amonio a partir de urea y arginina (Burne \& Marquis, 2000), lo que provee un mecanismo para equilibrar la producción de ácido desde el azúcar ingerido de la dieta, manteniendo así el equilibrio desmineralización/ remineralización. Sin embargo, cuando los hidratos de carbono se suministran con frecuencia, la acidificación es moderada, lo que permite que las bacterias no mutans se adapten al medio ácido, produciendo en el tiempo un desequilibrio entre la desmineralización/remineralización, inclinándose hacia la pérdida de minerales que conduce al inicio y progresión de la enfermedad de caries dental (Wescombe et al., 2009). 
Bajo prolongadas condiciones ácidas, las bacterias se vuelven más acidúricas, en esta etapa. Los S. mutans y Lactobacilos así como cepas acidúricas de Streptococcus no mutans, Actinomyces, Bifidobacterias y levaduras pueden llegar a ser dominantes, entre muchas otras bacterias acidógenas y acidúricas. La acidificación del medio ambiente es el principal determinante de los cambios fenotípicos y genotípicos que se producen en la microflora durante la caries (Takahashi \& Nyvad, 2011).

En la etapa inicial de la caries (lesión en esmalte) hay un aumento de bacterias tolerantes al estrés ácido y fermentadoras de azúcar, por lo que los determinantes de esta etapa son el $\mathrm{pH}$ y la dieta. Sin embargo, en lesiones en dentina hay un aumento de bacterias tolerantes al stress osmótico con actividad colagenasa y proteasa, permitiéndose así la degradación de la dentina, por lo que los determinantes de esta etapa son las bacterias acidogénicas y las bacterias proteolíticas. A partir de esto se ha propuesto que la enfermedad de caries es un proceso en el que las bacterias productoras de ácido son el vehículo para la entrada y penetración del esmalte, permitiendo que las bacterias degradadoras de dentina expandan la cavidad. Por lo que se sugiere que la diversidad bacteriana de la caries varía según su progresión, lo que es indicativo de que la flora bacteriana asociada a la enfermedad es altamente compleja (Simón-Soro et al., 2013a).

Microbioma oral. La cavidad oral humana está fuertemente colonizada por microorganismos, incluyendo virus, protozoos, hongos, bacterias y arqueas. En contraste con la microbiota que se encuentra en otras partes del cuerpo, que típicamente viven en armonía con el anfitrión, la microbiota normal de la boca es la responsable de las dos enfermedades más comunes del hombre, la caries y la enfermedad periodontal (Wade, 2013).

Las comunidades bacterianas que se encuentran en la boca son muy complejas con participación de alrededor de 1,000 especies (Dewhirst et al., 2010), siendo la segunda más compleja del cuerpo después del colon (Human Microbiome Project Consortium. Structure, function and diversity of the healthy human microbiome. Nature, 2012). Es necesario, por lo tanto, una combinación de análisis (filogenético, metagenómico, transcriptómico, proteómico y metabolómico) para diseccionar completamente las interacciones del microbioma oral, relevantes para la salud y enfermedad (Wade).
El conocimiento inicial de la composición del microbioma bacteriano oral tuvo su origen en la microscopía con las observaciones de Leeuwenhoek, quien mostró una colección de diversos morfotipos bacterianos en la placa dental (Wade). Tras el desarrollo de medios de cultivo bacteriano por Koch, Pasteur y otros, la microbiota cultivable de la boca formó una colección diversa de organismos, incluyendo aerobios estrictos, anaerobios facultativos y estrictos, con una amplia gama de potencial metabólico, incluyendo la capacidad de degradar azúcares, proteínas y sustratos complejos derivados de ellos. Este trabajo basado en el ambiente condujo a la identificación de organismos específicos que desempeñarían un papel causal en la caries dental y la periodontitis (Wade). Sin embargo, se ha descrito que una parte sustancial de la vida bacteriana en la tierra no puede ser cultivada en el laboratorio (Amann et al., 1995). En la cavidad oral, por ejemplo, aproximadamente la mitad de las bacterias presentes aún no se han cultivado (Wade). Por lo tanto, estas comunidades bacterianas complejas, han debido ser caracterizadas utilizando diferentes abordajes experimentales.

Métodos de estudio del microbioma oral. Análisis ARNr 16S. Este método se fundamenta en el análisis de secuencias conservadas de genes directamente aislados a partir de muestras de saliva o placa oral, siendo el gen más comúnmente usado para este propósito el que codifica el ARNr 16S (Wade). Esta molécula de ARN corresponde a una subunidad menor del ribosoma procariota, altamente conservado entre distintas especies bacterianas debido a que tiene una tasa de mutación baja, y que además, contiene secuencias hipervariables que proveen de secuencias específicas para cada especie, por lo que es usado como estándar para la clasificación e identificación de bacterias (Nyvad et al.), Uno de los métodos de estudio del ARNr $16 S$ es la electroforesis en geles denaturantes en gradiente, donde los genes ARNr 16S, con diferentes secuencias tendrán un comportamiento distinto al desnaturalizarse y su movilidad electroforética en el gel, será diferente. Las ventajas de este método es que es económico, fácil de realizar, confiable y excelente para el análisis inicial de la diversidad microbiana en saliva o placa oral. Por otra parte, tiene ciertas limitaciones, la técnica no es cuantitativa, tampoco es posible relacionar las bandas con especies conocidas, y es difícil su comparación con otros geles en muestras que contienen gran número de especies. Li et al. (2007) y Jiang et al. (2011) han utilizado esta técnica para el análisis de la composición de la placa en las diferentes etapas de la caries 
ASTORGA, B.; BARRAZA, C.; CASALS, J. M.; CISTERNA, M. J.; MENA, D.; MORALES, F.; GONZÁLEZ, S.; OLIVEIRA JUNIOR, O. \& MONCADA, G. Avances en el estudio de la diversidad bacteriana oral asociada a caries dental mediante el estudio genómico. Int. J. Odontostomat., 9(3):349-356, 2015.

dental en niños, demostrando que la riqueza y complejidad de especies microbianas disminuye durante el proceso de la caries.

Otro método de estudio del ARNr $16 \mathrm{~S}$ es el basado en la reacción en cadena de la polimerasa, PCR (Del inglés=Polymerase Chain Reaction), El análisis de muestras de material genético mediante PCR tiene como ventajas que es fácil de realizar y es un método confiable para la detección de patógenos cariogénicos. Como desventaja podemos mencionar que no evalúa otros organismos presentes en las muestras que no puedan ser conocidos por los partidores específicos utilizados. Existen métodos que permiten la evaluación de 3 a 4 patógenos en una reacción (Ciric et al., 2010), e incluso hay otros que permiten identificación de entre 9 a 20 microorganismos (Terefework et al., 2008; Pham et al., 2011), siendo de todas maneras insuficientes para conocer toda la diversidad bacteriana presente en la cavidad oral. Por otro lado, los múltiples ciclos de amplificación pueden afectar la abundancia de los diferentes grupos detectados, alterando la estructura de las comunidades bacterianas asociadas a salud y enfermedad (Xu \& Gunsolley, 2014). Sin embargo, como este método se basa en las regiones hipervariables del ARNr, algunas veces resulta difícil separar especies estrechamente relacionadas debido a la conservación de estos genes y a la alta complejidad de las especies bacterianas en la muestra (Xu \& Gunsolley). Usando este abordaje se ha demostrado la asociación entre $S$. Mutans y S. Sobrinus y caries temprana de la infancia (Choi et al., 2009; Palmer et al., 2010).

Una herramienta útil para la caracterización de comunidades bacterianas es el microarreglo de ARNr 16S. Los microarreglos taxonómicos se han desarrollado para los diferentes tipos de muestras ambientales, donde cada sonda de un gen ARNr $16 S$ es complementaria al de la especie objetivo (blanco). Los genes 16S RNAr presentes en la comunidad microbiana oral son amplificados por PCR, marcados e hibridizados en el microarreglo, por lo tanto este método se puede utilizar para identificar las comunidades bacterianas y conocer la asociación con infecciones orales u otras enfermedades (Call et al., 2003). Es así como se ha demostrado que la diversidad bacteriana es mayor en individuos sin lesiones de caries, en comparación con individuos con lesiones de caries radiculares y en dentina, donde el Actinomyces y el $S$. mutans parecieran estar jugando un rol limitado como patógenos de las lesiones de caries radicular (Preza et al., 2009). El uso de esta metodología, per- mitió al Instituto Forsyth desarrollar el HOMIM (Human Oral Microbe Identification Microarray), base de datos de microarreglos taxonómicos capaz de detectar aproximadamente 300 de las bacterias orales más prevalentes, incluidas algunas que no han podido ser cultivadas. Este método es cuantitativo y las muestras pueden ser dirigidas específicamente a pacientes individuales. La principal desventaja de este método, es que sólo identifica y analiza las bacterias para las cuales se haya preparado el microarreglo taxonómico, no identificando el total de bacterias en la muestra (Nyvad et al.), por lo que no es considerado un método ilimitado ("open-ended"), a diferencia del pirosecuenciamiento u otros similares.

Para evitar los sesgos inherentes a la aplicación del PCR por sí sola o acoplada a clonamiento y posterior secuenciación, es que se han desarrollado métodos de secuenciamiento de última generación (Next Generation Sequencing= NGS), entre los cuales tenemos: 454 pirosecuenciamiento, Illumina HiSeq/ MiSeq, ABISOLiD y Ion Torrent semiconductor sequencers. Estos análisis permitieron identificar un número mayor de especies bacterianas, y que estas son diferentes dependiendo del sitio de la cavidad oral desde donde se obtuvo la muestra (Aas et al., 2005).

Los métodos NGS permiten el secuenciamiento directo del ADN total de una comunidad de bacterias. Las principales ventajas de esta metodología son su alto rendimiento y que no requieren clonamiento, lo que simplifica el procedimiento. Utilizando esta nueva metodología y con el fin de describir el metagenoma oral en condiciones de salud y enfermedad, BeldaFerre et al., estudiaron muestras de placa bacteriana de la cavidad oral de individuos sanos y con enfermedad de caries, catergorizándolos en libres de caries, tratados por caries y con caries activa. Se tomaron muestras de la placa supragingival de los pacientes y en el caso de pacientes en actividad de caries se tomó una muestra de placa cercana a la lesión y además una muestra de la lesión misma. A pesar del bajo número de muestras examinadas, 25 en total, se encontraron diferencias interesantes en cuanto a la diversidad bacteriana entre individuos sanos y enfermos. En individuos sanos se encontró tendencia a una mayor población de bacilos y Gamma-proteobacterias, mientras que grupos anaerobios son más frecuentes en sujetos enfermos, como los Clostridios y los Bacteroidetes. Los bacilos están particularmente ausentes en las lesiones de caries, y una de las muestras presentó grandes proporciones de Actinobacteria. También se encontró al Aggregatibacter y al 
ASTORGA, B.; BARRAZA, C.; CASALS, J. M.; CISTERNA, M. J.; MENA, D.; MORALES, F.; GONZÁLEZ, S.; OLIVEIRA JUNIOR, O. \& MONCADA, G. Avances en el estudio de la diversidad bacteriana oral asociada a caries dental mediante el estudio genómico. Int. J. Odontostomat., 9(3):349-356, 2015.

Streptococcus sanguis como uno de los grupos bacterianos más prevalentes en sujetos libres de caries, y se identificó al Streptococcus gordonii y Leptotrihia buccalis como las bacterias más abundantes en sujetos cursando enfermedad de caries. Todo esto sugiere que a pesar que la diversidad bacteriana es muy amplia en la cavidad oral, muy pocos grupos taxonómicos de bacterias representan las grandes proporciones de la microflora oral (Belda-Ferre et al.).

Posteriormente, con el fin de estudiar si la diversidad bacteriana de diferentes sitios de la cavidad oral es representativa de la biodiversidad bacteriana oral, es que Simon-Soro et al. (2013a) analizaron muestras de la superficie bucal y lingual de los dientes y surcos gingivales, dorso de lengua y saliva de dos voluntarios sanos, a través de PCR y pirosecuenciamiento. La información genética recopilada de las muestras se analizó y cuantificó en base a Unidades Taxonómicas Operacionales (Operational Taxonomic Units - OTUs), donde cada OTU corresponde a secuencias de nucleótidos de 16S de ARNr agrupadas, las cuales tienen un $97 \%$ de similitud entre sí, las cuales permiten estimar un número aproximado de especies bacterianas presentes en la muestra, considerando 1 OTU como una especie bacteriana (Yarza et al., 2008). Los autores muestran diferencias en la composición y diversidad bacteriana entre los diferentes sitios de la cavidad oral, un hecho que es relevante a la hora de la elección del sitio de toma de muestra. Las muestras de saliva y lengua presentaron un mayor número de OTUs con respecto a dientes y surcos gingivales. La superficie lingual de los surcos gingivales mostró el nivel más bajo de OTUs. En las muestras de superficies dentarias y surcos gingivales se encontraron dentro de las especies más representativas los Streptococcus, Veillonella y Fusobacterium. Además se observaron diferencias significativas entre las muestras analizadas de la zona bucal del surco gingival donde predominó el Streptococcus y de la zona lingual del surco gingival donde predominó el Fusobacterium considerablemente sobre el Streptococcus. En superficie dental bucal, los Streptococcus se encontraron entre un $29-70 \%$ del total de especies bacterianas, en la superficie bucal surco gingival en un $23-57 \%$, en lingual de la superficie dental de un $0-5 \%$ y en lingual de surco gingival de un $5-21 \%$. El amplio rango de estos resultados puede deberse al reducido número de individuos de los cuales se recolectaron las muestras sin embargo, es importante señalar que es el primer trabajo que no utiliza un "pool" de muestra de diferentes sitios, sino que los analiza por separado poniendo en evidencia la diversidad según nicho ecológico analizado (SimónSoro et al., 2013a). Considerando este importante hecho, Simon-Soro et al. (2013b) evaluaron la composición bacteriana comparando individuos sanos versus individuos con enfermedad de caries. Analizaron ADN de muestras de placa dentaria de superficie de esmalte intacto, lesiones de mancha blanca en esmalte y caries dentinaria, y observaron que en esmalte intacto se encontraron 1015 OTUs, en lesiones de mancha blanca en esmalte 193 OTUs y en caries dentinaria profunda 290 OTUs. También se observaron que en las lesiones de esmalte las bacterias $S$. mutans, $S$. gordonii, Neisseria, Fusobacterium y Capnocytophaga eran más predominantes. Adicionalmente en las lesiones en dentina superficial, predominaba la familia del Streptococcus y Prevotella, y en lesiones de dentina profunda había un incremento de Lactobacilos, indicando que esta especie no juega un rol central en la iniciación de caries pero si en la progresión de la lesión. En general la cantidad de Streptococcus aumenta a medida que progresa la lesión. Sin embargo, esto no sucede con el S. mutans, que aumenta su proporción en las lesiones de caries, pero aparece en una baja frecuencia y tiende a disminuir a medida que la lesión avanza hacia la dentina (Simón-Soro et al., 2013a).

Como sabemos, el NGS es un proceso más rápido que otros métodos de secuenciamiento y permite analizar una gran cantidad de genes, sin embargo, al ser la última herramienta descrita para análisis genómico aún los softwares se encuentran en desarrollo para la identificación de tan alto número de genes, con el fin de disminuir los errores en la identificación. Las secuencias derivadas pueden ser muy cortas para la identificación a nivel de especies, aunque se puede facilitar la identificación si se compara con muestras específicas de ciertas bacterias. Además presenta ciertas limitaciones, ya que no toma como referencia el fenotipo de la bacteria, ya que la identificación de información genética similar no significa que el fenotipo sea idéntico, y en el proceso de enfermedad y particularmente en el desarrollo de caries la identificación del fenotipo es fundamental. Por esta razón, los nuevos estudios acuñan los conceptos de metaproteoma, metatranscriptoma, y metaboloma para una completa identificación y descripción de las comunidades bacterianas (Nyvad et al.).

Es importante señalar, que por la gran heterogeneidad que poseen los patógenos orales, no se puede confiar solo en la secuencia del 16S RNAr para comprender el grado de cariogenicidad que estos po- 
seen (Gross et al., 2010), ya que tanto el S. mutans, como otros Streptococcus y bacterias de la placa dental, difieren en las características de adherencia, formación de la biopelícula, acidogénesis y la tolerancia al ácido. Todos lo anterior tiene un impacto directo en su capacidad para contribuir en el proceso de caries (Burne et al, 2012). Otra razón de porque la secuencia de 16S RNAr es insuficiente, es el concepto de plasticidad fenotípica. Este se refiere a que las bacterias crecen en función del ambiente en que se encuentran, por lo que el fenotipo puede ser muy diferente (Lemos \& Burne, 2008). Los factores ambientales, incluyendo el pH, reacciones oxido-reducción, fuente de hidratos de carbono, densidad de la población y la fase de crecimiento de las bacterias, tiene un gran impacto en la expresión genética de muchos estreptococos orales e influyen en los fenotipos que están directamente relacionados con la cariogenicidad (Burne et al., 2009).

Todos estos métodos tienen por objetivo identificar la información genética para así conformar un metagenoma bacteriano de la cavidad oral, es decir, conjunto de genomas bacterianos que permiten asociar características taxonómicas de las bacterias. Conocer la diversidad microbiana oral otorga información relevante para la actualización de los conocimientos y con esto es posible apuntar hacia nuevos objetivos terapéuticos.

\section{CONCLUSIONES}

La diversidad microbiana de la cavidad oral es amplia y diversas técnicas se han desarrollado con el objetivo de cuantificar e identificar la población en salud y enfermedad. Gracias al estudio del metagenoma de la cavidad oral, se ha observado que la composición de dicha población bacteriana varía significantemente cuando el hospedero manifiesta actividad de caries o al cambiar sus hábitos alimenticios y/o higiene.

El S. mutans es el microorganismo más estudiado en investigaciones sobre la caries dental y es un estándar para medir riesgo cariogénico en los individuos debido a su participación en la enfermedad y su marcada capacidad acidogénica y acidúrica, necesarios para el desarrollo de la lesión de caries. Sin embargo, esto no significa que sea un indicador certero sobre el riesgo y desarrollo de la enfermedad debido a que participan un gran número de otros microorganismos asociados a este proceso, especialmente si se considera que cada especie no tiene un rol determinado, sino que son heterogéneos, con alta variabilidad genética y fenotípica. Esta es una limitación importante en los estudios genómicos del microbioma oral debido a que en el desarrollo de caries influye de gran manera el metabolismo y rol de cada microrganismo.

La herramienta del metagenoma permite identificar especies que no han podido ser aisladas por métodos convencionales, además de identificar su presencia o ausencia en las distintas etapas del desarrollo de la enfermedad de caries dental, que permite un mejor conocimiento del desarrollo de esta.

Si bien es cierto que con el gen 16S RNAr, comúnmente utilizado en los métodos tradicionales de análisis microbiológico, se ha avanzado enormemente en el conocimiento sobre la diversidad microbiana oral, permitiendo comparar la microbiota oral en salud y enfermedad se debe tener en cuenta que el estudio únicamente de este gen presenta ciertas limitaciones.

Los avances en el conocimiento sobre la composición de las poblaciones bacterianas permitiría la identificación de los factores que la modifican favoreciendo el predominio de poblaciones bacterianas benéficas como nuevo foco terapéutico para pacientes en riesgo de actividad de la enfermedad de caries dental.

ASTORGA, B.; BARRAZA, C.; CASALS, J. M.; CISTERNA, M. J.; MENA, D.; MORALES, F.; GONZÁLEZ, S.; OLIVEIRA JUNIOR, O. \& MONCADA, G. Advances in the study of oral bacterial diversity associated with dental caries by genomic study. Int. J. Odontostomat., 9(3):349-356, 2015.

ABSTRACT: Dental caries is an infectious, transmissible and multifactorial disease, which leads towards a reversible and irreversible loss of minerals found in hard tissues of a tooth, caused by acids from carbohydrates fermentation due to metabolic activity of the biofilm attached to the tooth surface. Although Streptococcus mutans has been thought to be responsible for tooth decay, another bacterium named no mutans has been linked to the beginning, progression and activity of the disease in the enamel, dentine and cement. One of the methodologies put into practice to deepen the study of oral bacteria diversity related to carious cavities is oral metagenome. This methodology allows the study of whole bacterial groups by the analysis of DNA in a particular environment without the need of isolating and cultivating 
species, providing information about the taxonomical and phylogenetic diversity of these groups. There are different methods to study the bacterial diversity, including 16 S rRNA analysis through electrophoresis, PCR, microarrays, next generation sequence (NGS). The metagenome tool permits to recognize species that have not been able to be isolated by conventional methods. As well as identify its presence or absence in the different stages of the dental caries development, which allows a better understanding of development of the disease. The metagenome-based study has revealed an unexpected oral microbial diversity, giving information relevant to the updating of knowledge and identifies new therapeutic targets. The purpose of this review is to present the main results has brought the study of the metagenome on microbial diversity, applied specifically to the oral bacterial community in health and caries disease.

KEY WORDS: dental caries, metagenome, oral microbiome, 16S rRNA.

\section{REFERENCIAS BIBLIOGRÁFICAS}

Aas, J. A.; Paster, B. J.; Stokes, L. N.; Olsen, I. \& Dewhirst, F. E. Defining the normal bacterial flora of the oral cavity. J. Clin. Microbiol., 43(11):5721-32, 2005.

Aas, J. A.; Griffen, A. L.; Dardis, S. R.; Lee, A. M.; Olsen, I.; Dewhirst, F. E.; Leys, E. J. \& Paster, B. J. Bacteria of dental caries in primary and permanent teeth in children and young adults. J. Clin. Microbiol., 46(4):1407-17, 2008.

Amann, R. I.; Ludwig, W. \& Schleifer, K. H. Phylogenetic identification and in situ detection of individual microbial cells without cultivation. Microbiol. Rev., 59(1):143-69, 1995.

Belda-Ferre, P.; Alcaraz, L. D.; Cabrera-Rubio, R.; Romero, H.; Simón-Soro, A.; Miguel, P. A. \& Mira, A. The oral metagenome in health and disease. ISMEJ, 6(1):46-56, 2012.

Burne, R. A. \& Marquis, R. E. Alkali production by oral bacteria and protection against dental caries. FEMS Microbiol. Lett., 193(1):1-6, 2000.

Burne, R. A.; Zeng, L.; Ahn, S. J.; Palmer, S. R.; Liu, Y.; Lefebure T.; Stanhope, M. J. \& Nascimento, M. M. Progress dissecting the oral microbiome in caries and health. Adv. Dent. Res., 24(2):77-80, 2012.

Burne, R. A.; Ahn, S. J.; Wen, Z. T.; Zeng, L.; Lemos, J. A.; Abranches, J. \& Nascimento, M. Opportunities for disrupting cariogenic biofilms. Adv. Dent. Res., 21(1):1720, 2009.
Call, D. R.; Borucki, M. K. \& Loge, F. J. Detection of bacterial pathogens in environmental samples using DNA microarrays. J. Microbiol. Methods, 53(2):235-43, 2003.

Ciric, L.; Pratten, J.; Wilson, M. \& Spratt, D.A. Development of a novel multi-triplex qPCR method for the assessment of bacterial community structure in oral populations. Environ. Microbiol. Rep., 2(6):770-4, 2010.

Choi, E. J.; Lee, S. H. \& Kim, Y. J. Quantitative real-time polymerase chain reaction for Streptococcus mutans and Streptococcus sobrinus in dental plaque samples and its association with early childhood caries. Int. J. Paediatr. Dent., 19(2):141-7, 2009.

Dewhirst, F. E.; Chen, T.; Izard, J.; Paster, B. J.; Tanner, A. C.; Yu, W. H.; Lakshmanan, A. \& Wade, W. G. The human oral microbiome. J. Bacteriol., 192(19):500217, 2010.

Fontana, M.; Young, D. A.; Wolff, M. S.; Pitts, N. B. \& Longbottom, C. Defining dental caries for 2010 and beyond. Dent. Clin. North Am., 54(3):423-40, 2010.

Gross, E. L.; Leys, E. J.; Gasparovich, S. R.; Firestone, N. D.; Schwartzbaum, J. A.; Janies, D. A.; Asnani, K. \& Griffen, A. L. Bacterial $16 \mathrm{~S}$ sequence analysis of severe caries in young permanent teeth. J. Clin. Microbiol., 48(11):4121-8, 2010.

Hintze, H. Approximal caries prevalence in Danish recruits and progression of caries in the late teens: a retrospective radiographic study. Caries Res., 35(1):27-35, 2001.

Jiang, W.; Jiang, Y.; Li, C. \& Liang, J. Investigation of supragingival plaque microbiota in different caries status of Chinese preschool children by denaturing gradient gel electrophoresis. Microb. Ecol., 61(2):342-52, 2011.

Lemos, J. A. \& Burne, R. A. A model of efficiency: stress tolerance by Streptococcus mutans. Microbiology, 154(Pt. 11):3247-55, 2008.

Li, Y.; Ge, Y.; Saxena, D. \& Caufield, P. W. Genetic profiling of the oral microbiota associated with severe earlychildhood caries. J. Clin. Microbiol., 45(1):81-7, 2007.

Nyvad, B.; Crielaard, W.; Mira, A.; Takahashi, N. \& Beighton, D. Dental caries from a molecular microbiological perspective. Caries Res., 47(2):89102, 2013.

Palmer, C. A.; Kent, R. Jr.; Loo, C. Y.; Hughes, C. V.; Stutius, E.; Pradhan, N.; Dahlan, M.; Kanasi, E.; Arevalo Vasquez, S. S. \& Tanner, A. C. Diet and caries associated bacteria in severe early childhood caries. J. Dent. Res., 89(11):1224-9, 2010. 
Pham, L. C.; Hoogenkamp, M. A.; Exterkate, R. A.; Terefework, Z.; de Soet, J. J.; ten Cate, J. M.; Crielaard, W. \& Zaura, E. Effects of Lactobacillus rhamnosus GG on saliva-derived microcosms. Arch. Oral Biol., 56(2):13647, 2011.

Pitts, N. B. Monitoring of caries progression in permanent and primary posterior approximal enamel by bitewing radiography. Community Dent. Oral Epidemiol., 11(4):228-35, 1983.

Preza, D.; Olsen, I.; Willumsen, T.; Boches, S. K.; Cotton, S. L.; Grinde, B. \& Paster, B. J. Microarray analysis of the microflora of root caries in elderly. Eur. J. Clin. Microbiol. Infect. Dis., 28(5):509-17, 2009.

Seif, T. Cariología: prevención, diagnóstico y tratamiento contemporáneo de la caries dental. Bogotá, Actualidades Médico Odontológicas de Latinoamérica,1997. pp.35-7.

Shwartz, M.; Gröndahl, H. G.; Pliskin, J. S. \& Boffa, J. A longitudinal analysis from bite-wing radiographs of the rate of progression of approximal carious lesions through human dental enamel. Arch. Oral Biol., 29(7):529-36, 1984.

Simón-Soro, A.; Tomás, I.; Cabrera-Rubio, R.; Catalan, M. D.; Nyvad, B. \& Mira, A. Microbial geography of the oral cavity. J. Dent. Res., 92(7):616-21, 2013a.

Simón-Soro, A.; Belda-Ferre, P.; Cabrera-Rubio, R.; Alcaraz, L. D. \& Mira, A. A tissue-dependent hypothesis of dental caries. Caries Res., 47(6):591-600, 2013b.

Takahashi, N. \& Nyvad, B. The role of bacteria in the caries process: ecological perspectives. J. Dent. Res., 90(3):294-303, 2011.

Terefework, Z.; Pham, C. L.; Prosperi, A. C.; Entius, M. M.; Errami, A.; van Spanning, R. J.; Zaura, E.; Ten Cate, J. M. \& Crielaard, W. MLPA diagnostics of complex microbial communities: relative quantification of bacterial species in oral biofilms. J. Microbiol. Methods, 75(3):558-65, 2008.

Wade, W. G. The oral microbiome in health and disease. Pharmacol. Res., 69(1):137-43, 2013.

Wescombe, P. A.; Heng, N. C.; Burton, J. P.; Chilcott, C. N. \& Tagg, J. R. Streptococcal bacteriocins and the case for Streptococcus salivarius as model oral probiotics. Future Microbiol., 4(7):819-35, 2009.

Xu P. \& Gunsolley, J. Application of metagenomics in understanding oral health and disease. Virulence, 5(3):424-32, 2014.
Yarza, P.; Richter, M.; Peplies, J.; Euzeby, J.; Amann, R.; Schleifer, K. H.; Ludwig, W.; Glöckner, F. O. \& RossellóMóra, R. The All-Species Living Tree project: a 16S rRNAbased phylogenetic tree of all sequenced type strains. Syst. Appl. Microbiol., 31(4):241-50, 2008.

Dirección para Correspondencia:

Prof. Dr. Sergio Gonzalez

Cariología

Facultad de Odontología

Universidad Mayor

Alameda 2013

Santiago

CHILE

Email: sergio.gonzalez@mayor.cl

Recibido: 04-02-2015

Aceptado: 17-07-2015 